\title{
A PARSER COPING WITH SELF-REPAIRED JAPANESE UTTERANCES AND LARGE CORPUS-BASED EVALUATION
}

\author{
Yuji Sagawa Noboru Ohnishi Noboru Sugie \\ Dept. of Information Enginecring, Nagoya University, Japan
}

\section{INTRODUCTION}

Self-repair(Levelt 1988) is a repair of utterance by speaker him/herself. A human speaker makes self-repairs very frequently in spontaneous speech. (Blackmer and Mitton 1991) reported that self-repairs are made once every 4.8 seconds in dialogues taken from radio talk shows.

Self-repair is one kind of "permissible illformedness", that is a human listener can feel ill-formedness in it but he/she is able to recognize its intended meaning. Thus your partner does not need to interrupt dialogue.

How do you feel if your partner interrupts dialogue every 5 seconds to ask "What do you mean?" or so? You will give up dialogue or choose means of writing. Speaking without self-repair is the most difficult modality of natural language communication.

The goal of our work is to make a dialogue system coping with self-repaired utterances. In this paper we propose a parser called SERUP(SEIC-Repaired Utterance Parser), which plays a major part in understanding a sell-repaired utterance. That is, because our approach is to translate a self-repaired utterance (Ex.1) into a wellformed version that does not contain selfrepair (Ex.2) and parse the well-formed one, we do not need to change the subsequent processes.

[Ex.1] And from green left to pink, er, from blue left to pink (from (Levelt 1988))

[Ex.2] And from blue left to pink
SERUP uses some linguistic clues to translate utterances, those include a repetition, an unknown word and/or an isolated word. We describe how SERUP uses these clues.

To evaluate SFRUP, we analyze a large corpus that contains spontaneous dialogues over telephone. From the result, we estimate that SERUP works well with $88.1 \%$ of 1,082 self-repairs in the corpus.

\section{RELATED WORKS}

(Hindle 1983) and (Langer 1990) proposed parsers coping with self-repaired utterances. But they assumed that an interruption point has already been detected. Hindle thought prosodic cues can be used in detection, but it is not clear if they can always succeed. Langer thought editing expressions can be used, but they are not always used in selfrepair.

Recently, (Shriberg, Bear, and Dowding 1992) proposed a pattern matching method and used it in GIEMINI system(Dowding et al. 1993). This is similar to our method, but the corpus(MADCOW 1992) used is less spontancous than ours. (Subjects pressed a button to begin speaking to the system)

(Nakatani and Hirschberg 1993) proposed a speech-first method in which prosodic cues are used mainly. We also think prosodic cues are important. But we think people use linguistic cues mainly because they can understand self-repaired utterances in transcripts.

All these works are done on English. (Janger also treats Germany) Because there are many syntactic differences (e.g., left branching v.s. right branching), it is not 
clear if their approach is applicable to Japanese.

\section{OUTLINE OF SERUP}

Fig.1 shows the outline of SERUP.

Normal Parser is a parser that parses wellformed utterances. When Normal Parser fails to parse an utterance, the utterance is passed to SR-reconstructor that detects a self-repair in it and translates it into wellformed version. The translated utterance is returned to Normal Parser and parsed again.

Because an utterance can contain two or more self-repairs, translation is repeated until Normal Parser succeeds in parsing or translation fails. In the latter case, the utterance has another ill-formedness or self-repair that the SR-reconstructor cannot cope with.

There are two main problems in translation. One is to determine an interruption point, and the other is to determine a reparandum. If these two problems can be solved, then the process of translation is carried out as follows.

1. Remove editing expressions such as er, no, I mean.

2. Supersede the reparandum with repair part.

For more detail of SERUP, see (Sagawa, Ohnishi, and Sugie 1993).

\section{CLUES TO TRANSLATION}

In this chapter, we will describe a classification of self-repaired utterances. They are Inputs

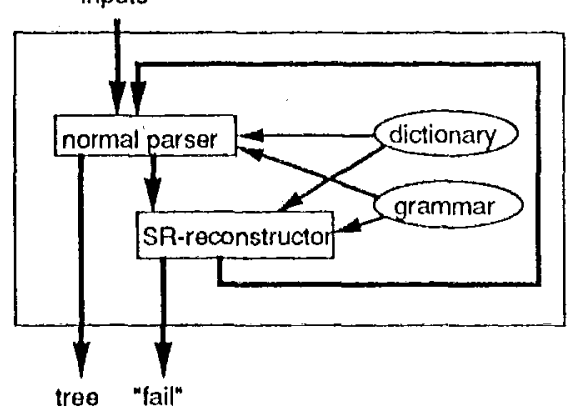

Fig. 1: The outline of SERUP classified by clues usable to determine an interruption point and a reparandum.

Table 1 shows the classification. Categories printed in italics have no clue, i.e., SERUP fails to parse utterances in those categories.

\section{with repetition}

A self-repair is mostly made in a way to repair a word or a phrase just before an interruption(Levelt 1988). So words or phrases around an interruption are in the same category. For example, in [Ex.1] speaker repairs a prepositional phrase "from green left to pink" to "from blue left to pink", It is rare that he/she just repairs a noun "green" to "blue".

In such self-repairs, a repetition of a word or a plurase often exists. In self-repairs which are intended to correct an error (such as [Ex.1]), words or phrases around the crror may be repeated.

In [Ex.1], "from" and "left to pink" are repeated. In self-repairs which are intended to add some information to the item just mentioned, the item may be repeated as in [Ex.3].

[Ex.3] I want a fight, one way flight (from (Shriberg, Bear, and Dowding 1992))

In this example a word "flight" is repeated. $\Lambda$ repetition is made with the same constituent or an itcm in the same category, such as "orange" with "apple".

There are four possible structures around an interruption of a self-repair with a repetition. Fig.2 shows them.

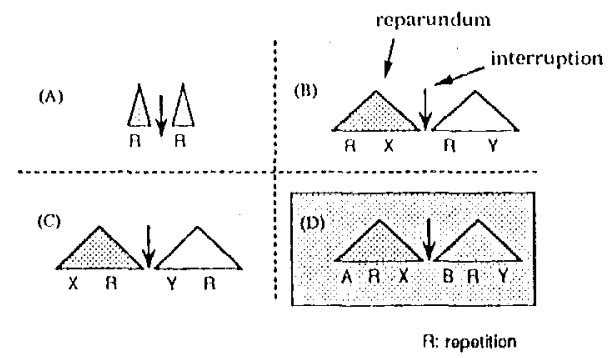

Fig. 2: Possible structures around interruption 
$\Lambda$ is a case of a simple repetition. B, C and $I)$ are cases in which some words exist between repetition. With cases $B$ and $\mathrm{C}$, positions of repetition directory indicate where an interruption occurs and which is a reparandum, but with $\mathrm{D}$ case, do not.

SERUP can cope with cases $\Lambda, B$ and $C$.

\section{with syntactic break}

A self-repair comes with an interruption of utterance. Because an interruption may occur anywhere in an ntterance (even within a word), self-repaired utterance can contain a syntactic break.

If this break can be detected, we can identify an interruption point.

\section{same fragment repetition}

When a speaker interrupts an utterance within a word, a fragment of the interrupted word is lelt. But he/she sometimes starts the repair with a word that begins with the same fragment as in [Fx.4].

\section{[Wx.4] ten, tenji tanntou no kata to}

'This can be treated as $\Lambda$ repetition, but to investigate a within-word intermution, wo treated it as a separate category.

In this case, an interruption point is just after a repeated fragment. And if withinword interruptions are only made to repair an interrupted word, a reparandum can be identified as the repeated fragment.

\section{with unknown word}

Sometimes a fragment left can be detected as an unknown word. For example, il a word "ketueki(blood)" is interrupted and a lragment "ketue" is left, this fragment can be detected because there is no Japanese word "ketue".

In this case, an interruption point is just after an unknown word. And the reparandum can be determined if the same condition as the above case is sufficed. with isolated word

$A$ fragment left by a within-word interruption is not always detected as the same lragment repetition or an unknown word. For example, a fragment "hon" can be left when "hontou"(real) is interrupted, but this string can be a word meaning "book".

But such a word is always "isolated", that is, both two subtrees in fig3 fail.

In this case, an interruption point is just after an isolated word. And reparandum can be determined if the same condition as the above case is sulficed.

\section{without repetition of a stem}

Because Japanese inflectional morphology is complicated, speakers often make inflection crrors. To repair such errors a speaker often starts a repair withont repetition of a stem ass in [Px.5] not as in [Ex.6].

[lix.5] itada $i$, kem no ka

[Fx.6] itada i, itada keru no ka

In these examples, "itada" is a stem and the speaker first tries to say "itada $i$ ta" or" "itada i te" and then changes to "itada kern".

In the case of [lix.6], a repetition of a stem can be used as a cluce. In the case of [lix.5], existence of an affix without a stem indicates an interruption point and a reparaudum.

\section{fresh start}

Fresh start is a repair with a completely different utterance. $A$ fragment of utterance belore intermption is ignored. SliRUP tries the detection of fresh start if all possible clues are not found. It tries to parse the fragment of utterance without a first word of it. It repeats this trial until parsing succeds.

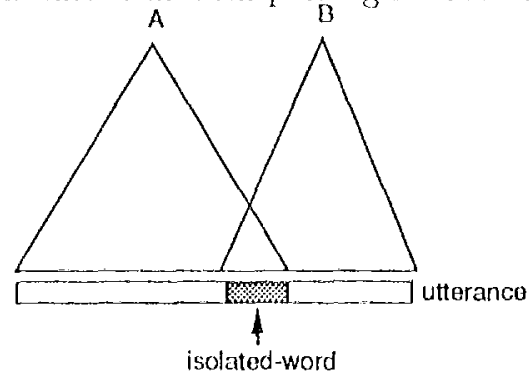

Fig. 3: An isolated word 


\section{others}

SERUP cannot cope with utterances of all these categories.

\section{changed to well-formed}

A self-repaired utterance is occasionally parsed successfully as a well-formed utterance that has a meaning that the speaker does not intend. For example, in [Ex.7], a fragment "kyou" of a word "kyousan "(cosponsorship) is treated as a word "kyou "(today), and parsed successfully but the meaning of it is "cosponsor today".

\section{[Ex.7] kyou, kyousan suru}

Some of these utterances can be detected as an error in semantic interpreter. And we think prosodic cues can be used effectively, because a fragment "kyou" and a word "kyou " is pronounced differently. So far, SERUP cannot cope with such utterances, because it uses well-formed first method.

\section{dividing word}

In [Ex.8] the speaker starts repair within word.

[Ex.8] junji, bi ni desu ne

The speaker tries to say "junbi ni desu ne", but makes a lexical error "junji". He starts the repair with a fragment "bi" of "junbi", instead of a complete word "junbi". This is a very rare case.

repetition with different category

Speakers occasionally repair with different category of words. A human listener can draw some inference and find relation between words, but automatic detection is difficult.

\section{ambiguous repair}

In [Ex.9], it is ambiguous what kind of selfrepair is made.

[Ex.9] apointo wo, ni, er, suuzitu tyuu ni
The speaker may repair a particle "wo" with "ni", or repair a fragment "ni" of a word "nisanniti" that has the same meaning of "suuzitu"(some days). We cannot solve this ambiguity automatically.

\section{LARGE CORPUS-BASED ANALYSIS}

To investigate effectiveness of SERUP we analyzed a large corpus called ADD(Ehara et al. 1990). ADD contains one million words of dialogues about registration to an international conference over telephone. ADD is created at ATR Interpreting Telephony Laboratories.

There are 1,082 self-repairs in the corpus. With these self-repairs, we investigate the categories they belong to. Table 1 shows the result.

\section{DISCUSSION}

In sum, SERUP scems to cope with $953(88.1 \%)$ of self-repairs. We think SERUP is effective to Japanese self-repaired utterances.

Most of utterances that SERUP cannot cope with are in the category "Changed to well-formed". As we mentioned, these utterances might be processed successfully with semantic constraints or prosodic cues. If we could implement them, SERUP would cope with $1,064(98.3 \%)$ sell-repairs.

\section{CONCLUDING REMARKS}

We proposed SERUP, a parser coping with self-repaired Japanese utterances. SERUP uses some linguistic clues and translates a self-repaired utterance into well-formed version and parses it. The result of large corpusbased analysis suggests that $88.1 \%$ of 1,082 self-repairs can be processed by SERUP.

Our future directions are to test the system with large grammar and lexicon and to incorporate prosodic processing. 
Table 1: The result of ananlsys

\begin{tabular}{|c|c|c|}
\hline \multirow{4}{*}{ With repetition } & A repetition & $\begin{aligned} \text { same constituent repetition } & 141(13.0 \%) \\
\text { same category repetition } & 108(10.0 \%)\end{aligned}$ \\
\hline & $\mathrm{B}$ repetition & $\begin{aligned} \text { same constituent repetition } & 96(8.9 \%) \\
\text { same category repetition } & 2(0.2 \%)\end{aligned}$ \\
\hline & C repetition & $\begin{aligned} & \text { same constituent repetition } 136(12.6 \%) \\
& \text { same category repetition } 3(0.3 \%) \\
&\end{aligned}$ \\
\hline & $D$ repetition & $\begin{array}{rr}\text { same constituent repetition } & 4(0.4 \%) \\
\text { same category repetition } & 0(0 \%)\end{array}$ \\
\hline With syntactic break & & $\begin{aligned} \text { Same fragment repetition } & 105(9.7 \%) \\
\text { With unknown word } & 98(9.1 \%) \\
\text { With isolated word } & 235(21.7 \%) \\
\text { Without repetition of a stem } & 23(2.1 \%) \\
\text { Fresh restart } & 6(0.6 \%)\end{aligned}$ \\
\hline Others & & $\begin{aligned} \text { Changed to woll-formed } & 111(10.3 \%) \\
\text { Dividing word } & 4(0.4 \%) \\
\text { etition with different category } & 5(0.5 \%) \\
\text { Ambiguous repair } & 4(0.4 \%)\end{aligned}$ \\
\hline Total of successable & & $953(88.1 \%)$ \\
\hline Total & & 1,082 \\
\hline
\end{tabular}

\section{References}

Blackmer, E. R. and J. L. Mitton (1991). Theories of monitoring and the timing of repairs in spontaneous speech. Cognition 39, 173-194.

Dowding, J. el al. (1993). Gemini: A natural language system for spoken-languliage understanding. In Proceedings of the 31st Annual Meeting of ACL.

Ehara, E. et al. (1990). Contents of the $\Lambda^{\prime} T R$ dialogue database. Technical Report TRI-0186, ATR Interpreting Telephony Research Laboratories.

Irindle, D. (1983). Deterministic parsing of syntactic non-fluencies. In Proceedings of the 21st Annual Conference of the ACI, pp. 123-128.

Langer, H. (1990). Syntactic normalization of spontaneous speech. In COLING 90, pp. 180-183.

Levelt, W. J. M. (1988). Speaking: From Intention to Articulation, Chapter 12, pp. 458-499. Cambridge, MA: The MIT Press.

MADCOW (1992). Multi-site data collection for a spoken language corpus. In Proccedings of the DARPA Speech and Natural Language Workshop, pp. 7-14.

Nakatani, K. and J. Ilirschberg (1993). A specch-first model for repair detection and correction. In Proceedings of the 31st Annual Meeling of $A C L$, pp. 46-53.

Sagawa, Y., N. Olınishi, and N. Sugie (1993). Repairing self-repairs in Japanese. In Procecdings of Natural Language Processing Pacific Rim Symposium (NLPRS '03), Fukuoka, pp. 191-198.

Shriberg, E., J. Bear, and J. Dowding (1992). Automatic detection and correction of repairs in human-computer dialog. In Proceedings of the DARPA Speech and Natural Language Workshop, pp. 419-424. 